\title{
Medial capsulo-ligamentous complex plasty of the second metatarsophalangeal joint in the treatment of static foot deformities
}

\author{
S.M. Gudy, V.V. Epishin, I.A. Pakhomov, V.V. Kuznetsov, A.G. Samokhin, S.O. Drantusov
}

Tsivyan Novosibirsk Research Institute Of Traumatology And Orthopedics, Novosibirsk, Russian Federation

\begin{abstract}
Introduction Improving the approaches to treating patients with hammer toe deformity is a relevant task. The problems of its management are a lack of uniform approaches to treatment, a significant number of complications and low patient satisfaction with the results of treatment. A frequent complication of its surgical treatment is a residual abduction deformity of the second toe. However, the available literature does not adequately cover the treatment of this pathology, and the methods of surgical correction have serious shortcomings. In this regard, the aim of the study was to evaluate the efficiency of the original method for correcting the abduction deformity of the second toe. The proposed approach is based on strengthening the medial capsuloligamentous structures of the metatarsophalangeal joint with the tendon of the brevis extensor digitorum muscle. Material and methods Treatment of 26 (100\%) patients operated for abduction deformity of the second toe at the Novosibirsk Research Institute of Traumatology and Orthopedics in 2016 and 2017 was analyzed. The index group included 15 (58 \%) patients in whom the residual deformity was corrected according to the method proposed. In the comparison group of 11 (42\%) patients, the capsulotomy method of the second metatarsophalangeal joint with transarticular Kirschner wire fixation was used. The study utilised clinical, radiological, surgical, functional and statistical methods. Final treatment results were evaluated one year after the operations. Results The use of the traditional method of abduction deformity correction in the comparison group demonstrated its extremely low efficiency. On the other hand, in the index group there were 13 (86.6 \%) good results, one (6.7 \%) was fair and one (6.7 \%) was poor. Discussion Many authors state the importance of restoring the capsulo-ligamentous complex of the metatarsophalangeal joint while correcting the toe deformity in the plantar plane. The method proposed by us enables to avoid residual deformities due to a number of technical merits with good clinical and functional results.
\end{abstract}

Keywords: foot, abducted toe deformity, metatarsophalangeal joint, extensor digitorum brevis, tendon transfer, capsuloligamentous plasty

\section{INTRODUCTION}

Deformity of lesser toes in the transverse plane is defined as the medial or lateral deviation of the longitudinal axis of the proximal phalanx with respect to the longitudinal axis of the metatarsal bone according to clinical and radiological data [1]. The lateral deviation of the second toe is known in the literature under various names that are synonymous terms: "hallux valgus deviation of the second toe", the syndrome of "pre-dislocation or instability of the second metatarsophalangeal joint in the plantar plane”, etc., but from the practical point of view, the term "abduction deformity of the second toe" (hereinafter ADST) is more suitable. This deformity causes a gross cosmetic defect and severe functional impairment of the forefoot. It has been proven that when the second toe is abducted, the first one loses its lateral support, which contributes to the lateral deviation of the big toe and may cause hallux valgus recurrence after reconstructive operations [2, 3]. Although deformities of the lesser toes in the plantar plane have been known for a long time, the etiology of ADST has not been precisely defined and, probably, it is multifactorial.

An important pathogenetic factor is the absence of the medial lumbrical muscle of the second metatarsophalangeal joint [4]. The damage to the stabilizers of the metatarsophalangeal joint (capsule, collateral ligaments and/or the plantar plate) as well as the imbalance in the traction force of the tendons passing around the joint (long and short flexors and extensors of the toes, lumbrical and interosseous muscles) are significant factors [5]. Moreover, it is necessary to emphasize a frequent iatrogenic role in the formation of residual abduction deformity due to the complex regional anatomy of the metatarsophalangeal joints of the foot and the presence of numerous anatomical structures in a limited space and, most importantly, the imperfection of the available treatment methods [6, 7].

It is not difficult to diagnose ADST basing on the findings of clinical examination and evaluation

Gudy S.M., Epishin V.V., Pakhomov I.A., Kuznetsov V.V., Samokhin A.G., Drantusov S.O. Medial capsulo-ligamentous complex plasty of the second metatarsophalangeal joint in the treatment of static foot deformities. Genij Ortopedii, 2019, vol. 25, no 4, pp. 468-473. DOI 10.18019/1028-4427-2019-25-4-468-473. (In Russian) 
of the radiographs of the foot in $\mathrm{AD}$ and lateral views taken under load [1]. The available methods of conservative treatment for ADST do not provide deformity correction and are aimed at alleviating the symptoms and eliminating the pathological conflict of the deformed toe with the shoes [8]. Surgical treatment is the method of choice, and various surgical options have been described, from simple percutaneous capsulotomy to comprehensive restoration of the involved stabilizing structures of the metatarsophalangeal joint with or without bone osteotomies. The choice of a specific correction technique should be based on the etiology, grade and mobility. Moreover, in the professional literature on foot surgery, the issues of choosing the ADST correction method have not been covered sufficiently. Thus, the goal of this work was to evaluate the effect of a new ADST correction approach.

\section{MATERIAL AND METHODS}

Surgical treatment of 26 patients with ADST (one (3.8 \%) male, 25 (96.2 \%) females) in the average age of $56 \pm 3.8$ years who underwent interventions at our institute in 2016 and 2017 was analyzed retrospectively. All patients had a history of previous operations for longitudinal and transverse flatfoot, hallux valgus of grade 3 and hammer deformity of the second toe. As the main stage of hallux valgus correction, all patients had undergone the Lapidus surgery, internal fixation. Correction of the first ray of the foot was achieved in all of them.

Arthrodesis of the proximal interphalangeal joint using an internal fixator combined with plantar plasty of the metatarsophalangeal joint to correct the hammer deformity of the second toe had been used in ten female patients (38.5 \%) in the average age of $50 \pm 7.3$ years, and the Taylor operation, arthrodesis of the proximal interphalangeal joint with percutaneous transarticular fixation with a Kirschner wire, had been performed in 16 patients (61.5\%), one male (6.3\%) and 15 females (93.7\%) among them in the average age of $58 \pm 2.7$ years [9].

Six months after the operations, a residual abduction deformity of the second toe was revealed in all the patients. The patients were re-hospitalized for surgical treatment for ADST. Patients were divided into two groups (index and control) by the method of ADST residual deformity correction. The comparison (control) group included 11 (42\%) patients in whom the method of capsulotomy of the second metatarsophalangeal joint with transarticular fixation of the toe with a Kirschner wire was used [1, 10]. The index group of 15 (58 \%) patients underwent ADST correction according to the original technique proposed by our clinic. Its methodology is tendon plasty of the medial capsulo-ligamentous complex of the second metatarsophalangeal joint, for which RF patent No. 2668467 have been obtained [11].
Clinical examination All patients were examined for clinical signs of the pathology before surgical treatment and its correction, immediately after the interventions, four weeks and one year after them by visual examining, palpating, measuring (with a goniometer) of the range of passive and active movements in the second metatarsophalangeal joint and the values of angulation at the level of the metatarsophalangeal and proximal interphalangeal joint of the second toe. To assess the support function of the second toe and the stability of the second metatarsophalangeal joint, all patients underwent functional tests: a "paper grip" test [12] and a modified Lachman drawer test [13]. The integrated frequency of clinical outcomes was evaluated based on classification features (Table 1).

Radiographic method Weight-bearing AP and lateral radiographic views of the foot were taken before the operation and one year after the operation. Also, radiographic checks were done intraoperatively. Toassess the abduction deformity of the second toe, angulation was evaluated at the level of the metatarsophalangeal and proximal interphalangeal joints in the sagittal and frontal planes. The main indicator of ADST is the magnitude of the second metatarsophalangeal angle in the anteroposterior projection.

Surgical method All operations ran under spinal anesthesia with the patient in the supine position and a squeezing hemostatic tourniquet was used on the involved limb. A linear incision was made over the second ray of the foot, three to $4 \mathrm{~cm}$ long and centered over the metatarsophalangeal joint. The skin and underlying soft tissues were dissected by layers; next, tendons of the long and short extensors of the toe were exposed. Normally, the tendon of the latter is located in the lateral subluxation and abducts the toe laterally. The metatarsophalangeal joint was exposed and lateral release was performed by longitudinal dissection of the dorsal capsule and collateral ligaments. 
Treatment outcome clinical criteria

\begin{tabular}{|l|l|}
\hline Clinical outcome & \\
\hline \multirow{4}{*}{ Good } & - painless foot and regular footwear (AOFAS 75-100 points) \\
\cline { 2 - 3 } & - radiography confirms correction, no residual deformities \\
\cline { 2 - 3 } & - no complications \\
\cline { 2 - 3 } & - patient's satisfaction with functional and cosmetic outcomes \\
\hline \multirow{4}{*}{ Fair } & $\begin{array}{l}\text { - painless foot or mild pain combined with one of the following signs: failure of the toe to contact } \\
\text { the supporting surface or the need in additional orthopaedic product use (AOFAS 51-74 points) }\end{array}$ \\
\cline { 2 - 3 } & - radiography confirms correction, no residual deformities \\
\cline { 2 - 3 } & - no complications \\
\cline { 2 - 3 } & - patient's satisfaction with functional and cosmetic outcomes \\
\hline \multirow{4}{*}{ Poor } & - foot pain, deformity recurrence, need to wear orthopaedic shoes (AOFAS 50 or less points) \\
\cline { 2 - 3 } & - radiography confirms insufficient correction, residual deformity and/or bone dislocation \\
\cline { 2 - 3 } & - complications such as suppuration, vascular or neurologic deficit, etc \\
\cline { 2 - 3 } & -patient's dissatisfaction with functional and cosmetic outcomes \\
\hline
\end{tabular}

Note: AOFAS scale for forefoot functional assessment

The second toe was fixed in the anatomical position with a transarticular Kirschner wire in the comparison group. The patients of the study group underwent tendon transfer of the short extensor of the toes of the foot, for which a canal was drilled with a two-millimeter tip burr perpendicular to the axis of the toe in the mediolateral direction at the base of the proximal phalanx. The tendon of the short extensor of the toes of the foot was cut off in its tendon-to-muscle portion. The distal end of the cut-off tendon was stitched with the "000" thread and was transferred through the canal formed to the medial surface of the base of the proximal phalanx. In the intertarsal space, the "thrown through" tendon was pulled under the complex of the tarsal ligaments and the deformed toe was reduced by tendon traction (Fig. 1). The physiological position achieved was secured by transossal fixation of the tendon of the short extensor to the diaphysis of the second metatarsal bone. Pulling the tendon between the heads of the metatarsal bones in the space provides reduction, and a strong fixation to the metatarsal bone diaphysis provides reduction stability.

Next, there was a radiographic control followed by wound stitching by layers, aseptic dressing, and "figured" bandaging. In the post-operative period, the patients were allowed a dosed weight-bearing on the foot in specialized unloading shoes for four weeks, followed by gradual inclusion of the anterior foot into weight-bearing. They all passed a rehabilitation programme under the supervision of a physiotherapist and exercise therapy instructor. The Kirchner wire in the control group patients was removed after four weeks.

Functional method Pain was assessed with a tenpoint VAS scale, and the AOFAS scale for the forefoot was used for functional parameters.

Statistical method Statistical processing was performed using the IBM SPSS v.21.0 software. The results of the study are presented by descriptive statistics. To assess the statistical significance of the differences in means, the Student's t-test and the Mann-Whitney nonparametric test were used. The significance level was 0.05 .
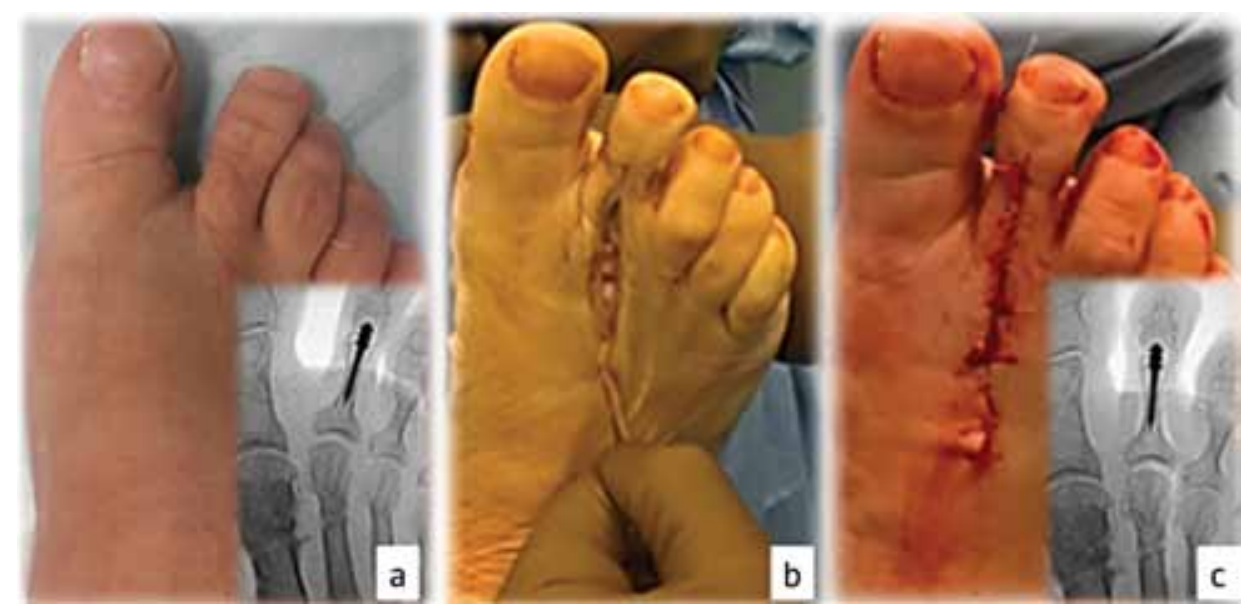

Fig. 1 Patient M., 49 years old, with residual abduction deformity of the second toe of the right foot with a second metatarsophalangeal angle of $26^{\circ}$ in the X-ray $(\boldsymbol{a})$; extensor brevis tendon transfer with the technique proposed and elimination of the second toe deformity by traction for the transferred tendon $(\boldsymbol{b})$; appearance of the foot after the operation; the second metatarsophalangeal angle is 4 degrees which is within the anatomical norm (c) 


\section{RESULTS}

Among 180 (100 \%) patients treated surgically for static forefoot deformities at the Novosibirsk Research Institute in 2016, ADST developed in 26 (14.4\%) patients. No correction effect was noted in 11 (42\%) patients of the comparison group of capsulotomy with transarticular fixation of the toe with a wire to correct ADST. All showed clinical and radiological manifestations of deformity recurrence (Table 2 ).

The method proposed by us and used in 15 (58 \%) patients of the index group was effective as correction and restoration of the function of the second toe were achieved (Table 2). Moreover, the range of passive and active movements in the second metatarsophalangeal joint one year after surgery corresponded to the preoperative values. The toe contacted the supporting surface in all 15 patients, as evidenced by a positive "paper grip" test. When comparing radiographs before the operation and one year after the operation, a significant decrease in the lateral deviation of the proximal phalanx to the average physiological norm was noted, and partial loss of correction was observed only in one patient (6.7 \%), which was associated with superficial infection in the post-operative period and gross degenerative changes in the soft tissues in the area of operation. All patients were satisfied with the outcomes and pain decreased on average by 2.5 points after the intervention. Functional indicators, according to the AOFAS scale for the forefoot, increased by 26.4 points at one-year follow-up.

Table 2

Dynamics of clinical and radiographic findings in the patients studied, $n=26(M \pm m)$

\begin{tabular}{|c|c|c|c|c|c|}
\hline \multirow{2}{*}{\multicolumn{2}{|c|}{ Parameter }} & \multicolumn{2}{|c|}{ Index group $(n=15)$} & \multicolumn{2}{|c|}{ Comparison group $(n=11)$} \\
\hline & & $\begin{array}{l}\text { Prior to } \\
\text { operation }\end{array}$ & $\begin{array}{l}\text { One-yea } \\
\text { follow-up }\end{array}$ & $\begin{array}{l}\text { Prior to } \\
\text { operation }\end{array}$ & $\begin{array}{l}\text { One-year } \\
\text { follow-up }\end{array}$ \\
\hline \multicolumn{2}{|c|}{ Passive movements in the second MPJ } & $61.3 \pm 9.3^{\circ}$ & $52.0 \pm 15.6^{\circ}$ & $59.4 \pm 11.3^{\circ}$ & $50.3 \pm 12.6^{\circ}$ \\
\hline \multicolumn{2}{|c|}{ Active movements in the second MPJ } & $33.2 \pm 13.7^{\circ}$ & $28.9 \pm 12.7^{\circ}$ & $30.3 \pm 17.8^{\circ}$ & $24.2 \pm 6.8^{\circ}$ \\
\hline \multicolumn{2}{|c|}{ Lateral deviation of the second toe } & $25.1 \pm 2.7^{\circ *}$ & $8.6 \pm 1.8^{\circ *}$ & $26.5 \pm 4.3^{\circ}$ & $18.3 \pm 6.7^{\circ}$ \\
\hline \multicolumn{6}{|c|}{ Functional tests (number of patients) } \\
\hline \multirow{2}{*}{ "Paper grip" test } & $\ll+»$ & 13 & 15 & 7 & 8 \\
\hline & «-» & 2 & - & 4 & 3 \\
\hline \multirow{3}{*}{$\begin{array}{l}\text { Lachman test } \\
\text { (Hamilton-Thompson, 1999) }\end{array}$} & 0 & 10 & 13 & 3 & 4 \\
\hline & $\mathrm{I}$ & 4 & 2 & 5 & 4 \\
\hline & II & 1 & - & 3 & 3 \\
\hline \multicolumn{6}{|c|}{ Radiographic findings in plantar plane } \\
\hline \multicolumn{2}{|c|}{ Second metatarsophalangeal angle } & $27,2 \pm 2,2^{\circ *}$ & $8,1 \pm 1,4^{\circ *}$ & $25,6 \pm 3,2^{\circ}$ & $20,3 \pm 2,7^{\circ}$ \\
\hline \multicolumn{2}{|l|}{ Proximal interphalangeal angle } & $3,1 \pm 1,5^{\circ}$ & $3,0 \pm 2,5^{\circ}$ & $1,1 \pm 2,3^{\circ}$ & $1,0 \pm 3,1^{\circ}$ \\
\hline \multicolumn{6}{|c|}{ Functional methods (points) } \\
\hline \multicolumn{2}{|l|}{ VAS scale } & 4.3 & 1.8 & 3.9 & 3.8 \\
\hline \multicolumn{2}{|l|}{ AOFAS scale } & 66.3 & 92.7 & 68.7 & 70.7 \\
\hline
\end{tabular}

Notes: * - statistical difference, $\mathrm{p}<0.5$; MPJ - metatarsophalangeal joint

\section{DISCUSSION}

Operations for static foot deformities, including hallux valgus and hammertoe deformity of lesser toes, are some of the most common orthopedic procedures. It is no secret that to date, there is a high rate of complications after them, one of which is ADST. Unfortunately, there is no sufficient information on the treatment of this complication in the professional literature. We conducted an analysis of the literature on ADST correction methods and found out that approaches to its treatment remain debatable. According to several authors, osteotomies for the correction of lesser toe deformities in the transverse plane have serious shortcominga along with their merits. Thus, the osteotomy proposed by W.H. Davis [14] of the proximal phalanx of the toe results in a residual subluxation or dislocation at the level of the metatarsophalangeal joint, which, according to the author, limits its use as a separate and/or main treatment option for ADST. The Weil osteotomy in the treatment of lesser toe deformities in the plantar plane ends with up to $35 \%$ of complications, as a number of authors report [15-18], which is an unacceptable risk level. The most common complication that occurs after the Weil osteotomy is a absent contact of the toe with the supporting surface under it or formation of the so-called "floating toe", aseptic necrosis of the osteotomised head, and bone fragmentation during osteosynthesis [19].

Thus, the current experience in the treatment of ADST shows the unambiguous need for interventions on the soft tissues of the metatarsophalangeal joint. The generally accepted method of capsuloligamentotomy 
with transarticular wire fixation, according to the results of our study and the literature [10], is low effective. ADST correction approach by restoring the damaged medial capsuloligamentous structures of the metatarsophalangeal joint is a pathogenetically substantiated approach. Anatomical restoration of the collateral ligament is extremely difficult due to the small amount of local tissues and the technical difficulties for manipulations in the narrow intermetatarsal space [1]. The method of choice for its restoration is tendon plasty. So, in 1992, J.T. Deland et al. [20] first proposed anatomical reconstruction of the metatarsophalangeal joint with restoration of the collateral ligament by a part of the tendon of the dorsal or plantar interosseous muscle. The merit of that study is that the authors used ten cadaver samples and confirmed that the collateral ligament plasty provides lateral stability and does not restrict movements in the metatarsophalangeal joint. However, the available literature does not report on the use of this method in clinical practice, and therefore it is impossible to judge its effectiveness. Further studies showed the possibility of using the tendon of the extensor brevis of the toes to restore the capsulo-ligamentous complex of the metatarsophalangeal joint without compromising the toe function [5, 21, 22, 23].

ADST correction methods similar ours have been described. In 2013, S.J. Ellis et al. [5] proposed an original technique for tendon transfer of the short extensor of the toes. In their operation, the tendon of the short extensor of the toe is cut off proximal to the level of the metatarsophalangeal joint and passed through the canal formed in the proximal phalanx in the direction from the medial dorsal to the lateral plantar side. Then it (the tendon) is transferred back through the second canal, formed in the direction from the lateral plantar to the medial dorsal side in the neck of the metatarsal bone, thus repeating the course and attachment points of the collateral ligament. Deformity of the toe is corrected by traction for the tendon; then tenodesis is performed with a screw with a diameter of 2.0 or $2.4 \mathrm{~mm}$ to the diaphysis of the metatarsal bone. However, although the method is aimed at reconstructing the anatomy of the medial collateral ligament considering its attachment points, the authors underestimate the importance of the interaction of collateral ligaments with the plantar capsulo-ligamentous complex, which probably causes the residual dorsal displacement of the toe corrected intraoperatively.

A similar biomechanical phenomenon of lesser toe deformity correction in the transverse plane by extensor brevis transfer was described by K.B. Hobizal et al. [23]. In the described method for conducting the short extensor tendon, the second toe may deviate dorsally, which is probably due to a subluxation of the proximal phalanx as a result of pulling the transferred tendon, which requires additional stabilization of the corrected toe with dressing bandages or "taping" in the early postoperative period. However, despite the residual deformities, the authors of both articles report high patient's satisfaction with the results of correction. The merit of the method developed is that when holding the tendon of the short extensor of the toes in the interspace, reducing traction is applied to the base of the proximal phalanx, and the congruence (relationship) of the articular surfaces is restored while strong fixation to the metatarsal diaphysis provides a stable reduction force and prevents deformity recurrence.

\section{CONCLUSION}

Analysis of the literature data on surgical treatment of lesser toe deformities in the transverse plane showed the ambiguity of the approaches to solving this clinical problem and the need to improve treatment methods for ADST. However, many authors stress the importance of restoring the capsuloligamentous complex of the metatarsophalangeal joints on the opposite side of the deformity. The method proposed by us avoids undesirable residual deformities due to a number of technical advantages. Thus, one-year follow-up in 15 (100\%) patients with abduction deformity of the second toe showed that our original method resulted in 13 (86.6\%) good, one $(6.7 \%)$ fair associated with residual pain in the metatarsophalangeal joint, and one $(6.7 \%)$ poor outcome associated with superficial infection, degenerative changes in the area of the surgical intervention and partial loss of deformity correction.

\section{REFERENCES}

1. Banks A.S., Downey M.S., Martin D.E., Miller S.J., eds. McGlamry's Comprehensive Textbook of Foot and Ankle Surgery. $3^{\text {rd }}$ Edition. Fundamental conditions and procedures. Section III. Philadelphia, Lippincott Williams \& Wilkins, 2001, vol. 1, pp. 202-224.

2. Hampar K. Hallux valgus, allied deformities of the forefoot and metatarsalgia. Philadelphia, Saunders, 1965, pp. 293-300.

3. Perera A.M., Mason L., Stephens M.M. The pathogenesis of hallux valgus. J. Bone Joint Surg. Am., 2011, vol. 93, no. 17, pp. 16501661. DOI: 10.2106/JBJS.H.01630.

4. Hur M.S., Kim J.H., Gil Y.C., Kim H.J., Lee K.S. New insights into the origin of the lumbrical muscles of the foot: tendinous slip 
of the flexor hallucis longus muscle. Surg. Radiol. Anat., 2015, vol. 37, no. 10, pp. 1161-1167. DOI: 10.1007/s00276-015-1488-6.

5. Ellis S.J., Young E., Endo Y., Do H., Deland J.T. Correction of multiplanar deformity of the second toe with metatarsophalangeal release and extensor brevis reconstruction. Foot Ankle Int., 2013, vol. 34, no. 6, pp. 792-799. DOI: 10.11 77/1071100713475433.

6. Gibbard K.W., T.E. Kilmartin. The Weil osteotomy for the treatment of painful plantar keratosis. Foot (Edinb.), 2003, vol. 13, no. 4, pp. 199-203. DOI: 10.1016/s0958-2592(03)00061-0.

7. Brodsky J.W., Passmore R.N., Shabat S. Transection of the plantar plate and the flexor digitorum longus tendon of the fourth toe as a complication of endoscopic treatment of interdigital neuroma. A case report. J. Bone Joint Surg. Am., 2004, vol. 86, no. 10, pp. $2299-2301$.

8. Banks A.S., Downey M.S., Martin D.E., Miller S.J., eds. McGlamry's Comprehensive Textbook of Foot and Ankle Surgery. $3^{\text {rd }}$ Edition. Compound deformities. Section V. Philadelphia, Lippincott Williams \& Wilkins, 2001, vol. 1, pp. 1095-1097.

9. Taylor R.G. An operative procedure for the treatment of hammer-toe and claw-toe. J. Bone Joint Surg., 1940, vol. 22, no. 3, pp. 608-609.

10.Downey M.S., McGlamry M.C., Spizzirri S.A. Transverse plane digital deformities. Banks A.S., Downey M.S., Martin D.E., Miller S.J., eds. McGlamry's Comprehensive Textbook of Foot and Ankle Surgery. $3^{\text {rd }}$ Edition. Philadelphia, Lippincott Williams \& Wilkins, 2001, vol. 1, pp. 354-371.

11.Pakhomov I.A., Gudi S.M. Sposob korrektsii otvedennoi deformatsii II-IV paltsev stopy [The way to correct the abduction deformity of II-IV foot toes]. Patent RF, no. 2668467, 2017. (in Russian).

12.Bouché R.T., Heit E.J. Combined plantar plate and hammer toe repair with flexor digitorum longus tendon transfer for chronic, severe sagittal plane instability of the lesser metatarsophalangeal joints: preliminary observations. J. Foot Ankle Surg., 2008 , vol. 47, no. 2, pp. 125-137. DOI: 10.1053/j.jfas.2007.12.008.

13.Coughlin M.J., Baumfeld D.S., Nery C. Second MTP joint instability: grading of the deformity and description of surgical repair of capsular insufficiency. Phys. Sportsmed., 2011, vol. 39, no. 3, pp. 132-141. DOI: 10.3810/psm.2011.09.1929.

14.Davis W.H., Anderson R.B., Thompson F.M., Hamilton W.G. Proximal phalanx basilar osteotomy for resistant angulation of the lesser toes. Foot Ankle Int., 1997, vol. 18, no. 2, pp. 103-104.

15.Beech I., Rees S., Tagoe M. A retrospective review of the Weil metatarsal osteotomy for lesser metatarsal deformities: an intermediate follow-up analysis. J. Foot Ankle Surg., 2005, vol. 44, no. 5, pp. 358-364.

16.Migues A., Slullitel G., Bilbao F., Carrasco M., Solari G. Floating-toe deformity as a complication of the Weil osteotomy. Foot Ankle Int., 2004, vol. 25, no. 9, pp. 609-613.

17.Trnka H.J., Gebhard C., Mühlbauer M., Ivanic G., Ritschl P. The Weil osteotomy for treatment of dislocated lesser metatarsophalangeal joints: good outcome in 21 patients with 42 osteotomies. Acta Orthop. Scand., 2002, vol. 73, no. 2, pp. 190-194.

18.Sharma D.K., Roy N., Shenolikar A. Weil Osteotomy of lesser metatarsals for metatarsalgia: a clinical and radiological follow-up. The Foot, 2005, vol. 15, no. 4, pp. 202-205. DOI: 10.1016/j.foot.2005.07.006.

19.Trnka H.J., Nyska M., Parks B.G., Myerson M.S. Dorsiflexion contracture after the Weil osteotomy: results of cadaver study and three-dimensional analysis. Foot Ankle Int., 2001, vol. 22, no. 1, pp. 47-50.

20.Deland J.T., Sobel M., Arnoczky S.P., Thompson F.M. Collateral ligament reconstruction of the unstable metatarsophalangeal joint: an in vitro study. Foot Ankle, 1992, vol. 13, no. 7, pp. 391-395.

21.Ruch J.A. Use of the EDB tendon for muscle-tendon balance of the lesser MPJ. Camastra C.A., Vickers N.S., Carter S.R., eds. Reconstructive Surgery of the Foot and Leg. Update'95, Podiatry Institute Publishing, Tucker, GApp., 1995, pp. 114-118.

22.Haddad S.L., Sabbagh R.C., Resch S., Myerson B., Myerson M.S. Results of flexor-to-extensor and extensor brevis tendon transfer for correction of the crossover second toe deformity. Foot Ankle Int., 1999, vol. 20, no. 12, pp. 781-788.

23.Hobizal K.B., Wukich D.K., Manway J. Extensor digitorum brevis transfer technique to correct multiplanar deformity of the lesser digits. Foot Ankle Spec., 2016, vol. 9, no. 3, pp. 252-257. DOI: 10.1177/1938640015617528.

Received: 06.03.2019

\section{Information about the authors:}

1. Sergey M. Gudy, M.D.,

Tsivyan Novosibirsk Research Institute Of Traumatology And Orthopedics, Novosibirsk, Russian Federation,

Email: Dr.Gydi@mail.ru

2.Vitaly V. Epishin, M.D.,

Tsivyan Novosibirsk Research Institute Of Traumatology And Orthopedics, Novosibirsk, Russian Federation,

Clinical Hospital № 81 of the Federal Medical-Biological Agency, Seversk, Russian Federation,

Email: vitvalep@mail.ru

3. Igor A. Pakhomov, M.D., Ph.D.,

Tsivyan Novosibirsk Research Institute Of Traumatology And Orthopedics, Novosibirsk, Russian Federation,

Email: pahomovigor@inbox.ru

4. Vasily V. Kuznetsov, M.D.,

Tsivyan Novosibirsk Research Institute Of Traumatology And Orthopedics, Novosibirsk, Russian Federation, Email: vkuznecovniito@gmail.com

5. Alexander G. Samokhin, M.D., Ph.D.,

Tsivyan Novosibirsk Research Institute Of Traumatology And Orthopedics, Novosibirsk, Russian Federation,

Email: motorist@inbox.ru

6. Semen O. Drantusov, M.D.,

Tsivyan Novosibirsk Research Institute Of Traumatology And Orthopedics, Novosibirsk, Russian Federation,

Email: drantusov93@mail.ru 